\title{
Riflessioni (provocatorie?) dopo la frequenza di alcuni incontri di Medicina Narrativa e non solo
}

Giornale di Tecniche Nefrologiche e Dialitiche 20I8, Vol. 30(3-4) 224-225

(C) The Author(s) 2019

Article reuse guidelines:

sagepub.com/journals-permissions

DOI: $10.1177 / 0394936218823522$

journals.sagepub.com/home/gtn

(S)AGE
Marco Lombardi

Editor in Chief Giornale di Tecniche Nefrologiche e Dialitiche

'Il tempo della comunicazione tra medico e paziente costituisce tempo di cura.'

Comma 8 dell'articolo 1 della legge 22 dicembre 2017, n.219.

Frequentare alcuni incontri/convegni ${ }^{1-5}$ di Medicina Narrativa (MN), ha fatto crescere in me l'esigenza di scrivere questo breve editoriale per la rubrica "Nefrologia Narrativa" (NN) di questo Giornale. In realtà, ho sentito anche l'esigenza di farmi socio SIMeN e ANED e di provare a frequentare un master di $\mathrm{MN}$ oltre che di leggere cercando di acculturarmi di più in materia.

Tutto questo ha fatto fluire nella mia mente il sogno di cambiare una nefrologia e dialisi (ND) in una ND-anchenarrativa, di proseguire con più energia nei prossimi anni con il concorso Quirino Maggiore, di stringere ancor di più la collaborazione con le associazioni di pazienti ed infermieri... per una lotta COMUNE, ed infine di cercare collaborazioni per la creazione di un ambulatorio nefrologico di MN.

No, non mi è rimasto 'cliccato' il tasto maiuscolo; COMUNE l'ho scritto volutamente in maiuscolo.

Cerco di spiegarmi. Seppur neofita, rispetto a molte persone che ho conosciuto in questi incontri, voglio esprimere qui alcune considerazioni che ritengo importanti.

Il lecito desiderio che la $\mathrm{MN}$ entri a far parte dello strumentario comune di medici, infermieri, tecnici, dietisti, ecc. non è cosa completa. Dovrebbe diventare una modalità conosciuta anche ai pazienti... ma io vado oltre perché non basta! Ecco il COMUNE: dovrebbe essere patrimonio di tutti, di tutte le persone che oggi sane, potrebbero un domani divenire malate.

Questo dovrebbe poter essere il vero obiettivo -secondo me- delle associazioni pro-MN.

Me lo fa pensare il fatto che la ricerca attuale dei veri esperti di MN è profilata verso la dimostrazione che la MN serve... a quello che deve servire. Quello che non mi vede in accordo con questa visione è il fatto che queste dimostrazioni non possano altro che essere prodotte tramite una ricerca basata sulle evidenze (evidence-based medicine, EBM). È anche giusto, certo, ma io nella mia dabbenaggine vi trovo una sorta di contraddizione: la narrative-based medicine (NBM) che si serve della EBM per referenziarsi!

In questi incontri ho capito che la MN cura l'anima, e la medicina tradizionale il corpo: entrambe curerebbero (si prenderebbero cura) della persona (fatta di anima e corpo). Che bisogno c'è di dimostrare una cosa così evidente $\mathrm{e}$ logica? La cura dell'anima (una cosa diversa per ognuno di noi) non è facilmente dimostrabile, né da dimostrare, anche proprio per l'unicità e la diversità della nostra anima da tutte le altre: come si fa a dimostrare quanto bene può fare -a chi lo riceve... e a chi lo dà- un abbraccio, una carezza, un ascolto vero, o un racconto?

Ammettiamo oggi d'essere alla MN-2.0 (ricordiamoci che la $\mathrm{MN}$ ha ormai radici lontane); la $\mathrm{MN}-3.0$ non dovrà avere -sempre secondo me- alcun bisogno di essere dimostrata in alcun modo.

Un'altra cosa che non trovo giusta (ma sicuramente sarò sommerso da appropriate e contrarie evidenze) è che ad un corso o master di MN possa iscriversi solo un laureato, un addetto ai lavori, insomma qualcuno che abbia una veste specifica. Ok, questa mia è una provocazione ma c'è, secondo me, un fondo di verità; se vogliamo che la MN si diffonda viralmente (come dovrebbe essere per non impiegarci i prossimi 50 anni) l'unica speranza è la 'cultura'. La cultura nello specifico si può fare con la formazione (leggasi alfabetizzazione). D'accordo una volta tanto con tutti: la formazione deve essere fatta più precocemente possibile, appena iscritti alle università, corsi, ecc.... ma attenzione qui si parla sempre e solo dei soliti addetti ai lavori!!! Certo sarebbe già molto ma... non abbastanza!!! La formazione ovvero la cultura della narrazione (una cosa che è insita in ognuno di noi... 
altrimenti non saremmo arrivati sino a qui ed ora... la Storia quella con la $\mathrm{S}$ maiuscola docet!, le favole e i racconti pure!) deve essere fatta a tutti, alle scuole medie e poi alle superiori e su, su sino ad arrivare a chi è specificamente preposto alla cura (prendersi cura) della salute.

Quando ho chiesto ad un vero professore di un master 'vorrei iscrivermi al tuo master assieme alla mia compagna (che non ha alcuna specificità per la cura della salute a parte la sua e di tutti quelli che le stanno vicino compreso il sottoscritto...e non è poco, ma ha grande buonsenso ed intelligenza), quando mi ha risposto 'no, lei non può' ho provato una profonda delusione... ed ho pensato 'cosi non andremo mai molto lontano'.

Ecco che mi riaffiora alla mente la frase di un grande dell'umanità: 'I have a dream' (e poi l'hanno fatto fuori!!).

Io senza essere grande, se non per l'età, ne avrei addirittura due, e ringrazio la MN per farmi sognare:

a. il primo più egoistico: umanizzare una UO di nefrologia e dialisi con la narrativa;

b. il secondo più generoso: sperare che si riesca a creare un Movimento (molto attinente alla politica... purtroppo?... serve!), che porti la narrativa alla conoscenza di tutti... per arrivare entro un decennio (?... mi piacerebbe vederlo) ad un qualcosa di veramente concreto e stabile.

Ma attenzione, per fortuna qualcosa si muove, e mi riferisco alla legge 219, alla recente sentenza del Tar del Lazio sulla illegittimità delle visite mediche a cronometro, ${ }^{6}$ e alla denuncia già presentata nel 2015 dal presidente della Società Italiana di Medicina Interna al loro $116^{\circ}$ Congresso Nazionale. ${ }^{7}$

Pertanto, sogniamo che ci farà un gran bene e buona lettura a tutti della rubrica di NN dove troverete anche 2 abstracts che abbiamo (vedasi) inviato alla SIMeN e che poi hanno trovato posto come presentazioni al recente congresso della SIN dedicato (se pur in minima parte) alla Bioetica ed alla Narrativa: qualcosa si muove? Speriamolo, e diamoci da fare!

\section{Dichiarazione di assenza di conflitto di interessi}

Gli Autori dichiarano di non avere conflitti di interessi.

\section{Finanziamenti}

Gli Autori dichiarano di non aver ricevuto finanziamenti specifici da qualsiasi ente nei settori pubblico, privato o senza fini di lucro.

\section{Referenze dei convegni/corsi frequentati e VB.}

1. La nuova responsabilità professionale in sanità: la legge Gelli-Bianco. Aula Magna della Agenzia per la formazione AUSL Toscana Centro, Sovigliana, 27 Marzo 2017.

2. Legge sul Biotestmento. Insiemepermano-Onlus, Hotel Adriatico, Firenze, 7 Febbraio 2018.

3. $2^{\circ}$ Congresso Nazionale della Società Italiana di Medicina Narrativa SIMeN. La complicità nella cura. Il racconto dei racconti. Arezzo, 10-12 Maggio 2018.

4. Essere, raccontare, esistere: la narrazione nel lavoro di cura. Riflessione ed esperienze intorno alla medicina narrativa. Il valore della Narrazione nelle Dialisi ed in Hospice dell'area territoriale di Pistoia. Ospedale S. Jacopo, Pistoia, 26 maggio 2018.

5. La legge 219/2017: norme in materia di consenso informato e di disposizioni anticipate di trattamento. Palazzo degli incontri, Firenze, 31 Maggio 2018.

6. Dott|Net, Medlex|Redazione: Accolto il Ricorso del Sindacato Sumai Contro la Regione Lazio. http://www .dottnet.it/articolo/24612/tar-illegittime-le-visite-mediche-a -cronometro-violano-il-giudizio/?tag=10568536171\&tkg=1 $\& \mathrm{cnt}=4$ DOTTNET, Accessed 31/05/2018

7. DottNet, Medlex/Redazione. Tar: illegittime le visite mediche a cronometro, violano il giudizio. http://www.quotidianosanita.it/lavoro-e-professioni/articolo.php?articolo_id=32103 DOTTNET, Accessed 31/05/2018 